\title{
Antibiotic profile of Pseudomonas aeruginosa strains isolated in a teritiary care centre
}

\author{
Sindhu Cugati ${ }^{1}$, Sharanya K ${ }^{2, *}$, Chitralekha Saikumar ${ }^{3}$ \\ ${ }^{1,2}$ Assistant Professor, ${ }^{3}$ Professor \& HOD, Dept. of Microbiology, Sree Balaji Medical College \& Hospital, Chennai, India
}

*Corresponding author:

Email: sharuisalways@yahoo.com

\begin{abstract}
Introduction: Multidrug resistance among clinical samples has emerged as a major problem in clinical settings. Pseudomonas aeruginosa is an opportunistic, nosocomial pathogen inherently resistant to many drugs and is able to acquire resistance to all effective antimicrobial drugs. Multidrug-resistant (MDR) P. aeruginosa is an emerging cause of mortality and morbidity which causes $4-60 \%$ nosocomial infections worldwide.

Aim: The aim of the present study was to determine the antimicrobial susceptibility pattern of $\mathrm{P}$. aeruginosa isolates from various clinical samples in a tertiary care centre.

Materials and Methods: 100 Pseudomonas aeruginosa isolates from various clinical samples, collected in a tertiary care hospital over a period of 6 months were included in the study. Antimicrobial susceptibility testing was performed using Kirby-Bauer disc diffusion method. Interpretation was done according to the CLSI guidelines. Quality control was performed by using a standard strain of Pseudomonas aeruginosa ATCC 27853.

Result: Among the entire gram negative organism isolated from different samples Pseudomonas aeruginosa isolates were around $29 \%$. Out of the 100 Pseudomonas aeruginosa isolates studied, $87 \%$ were sensitive to Imipenem, $69 \%$ to Amikacin, $82 \%$ to Piperacillin-Tazobactum, $69 \%$ to Aztreonam, $31 \%$ to third generation cephalosporins, $34 \%$ to Gentamicin \& $47 \%$ to Ciprofloxacin respectively

Conclusion: In order to control antimicrobial resistance and to keep it in check it is necessary to practice effective antibiotic policies, frequent surveillance programs and adequate infection control measures.
\end{abstract}

Keywords: Drug Resistance, Disc diffusion method, Pseudomonas aeruginosa.

\section{Introduction}

Multidrug resistance among clinical samples has emerged as a major problem in clinical settings. Regular screening of antimicrobial susceptibility pattern of various isolates on a regular basis can greatly contribute in reducing the prevalence \& spread of multidrug resistant strains. ${ }^{1}$ Pseudomonas aeruginosa (P. aeruginosa), a Gram-negative, aerobic rod belonging to family Pseudomonaceae is an opportunistic nosocomial pathogen., ${ }^{2,3}$ It is highly resistant to disinfectants \& can tolerate a variety of physical conditions. ${ }^{4,5} \mathrm{P}$. aeruginosa is found to be causing both hospital-acquired and communityacquired infections. ${ }^{6}$ It causes ventilator associated pneumonia, urinary tract infections in intensive care units and also causes infection in burns patients. ${ }^{7}$

According to CDC and the NNISS, P. aeruginosa is responsible for $(17 \%)$ nosocomial pneumonia and takes $2^{\text {nd }}$ place in being the common causative of it, $(7 \%)$ urinary tract infection and takes the third place as most common cause, surgical site infection $(8 \%)$ being the fourth most common cause, the seventh most frequently isolated pathogen from the bloodstream (2\%) and the fifth most common isolate (9\%) overall from all sites. ${ }^{8}$ Infections caused by P. aeruginosa are particularly difficult to treat because the organism is intrinsically resistant to many antibiotics and is able to acquire resistance to all effective antimicrobial drugs by mechanisms such as MDR, efflux pumps, biofilm formation, production of $\beta$-lactamases and aminoglycoside modifying enzymes. ${ }^{9,10}$ There is an increase in resistance to antipseudomonal antibiotics worldwide.

Multidrug-resistant (MDR) $P$. aeruginosa is an emerging cause of mortality and morbidity which causes $4-60 \%$ nosocomial infections worldwide. ${ }^{11}$ In Asia alone the multidrug resistant (MDR) rates of p.aeruginosa is found to be $42.8 \%$,Extensive drug resistance rate (XDR) is $4.9 \%$,pan drug resistance (PDR) is $0.7 \%$ among nosocomial pneumonia patients. ${ }^{12 .}$ Henceforth, this study was carried out, to estimate the prevalence and resistance pattern of $P$. aeruginosa isolates that would accelerate us to follow appropriate infection control practices.

\section{Materials and Methods}

100 Pseudomonas aeruginosa strains were isolated from various clinical samples such as pus, urine, blood, sputum, endotracheal aspirate, CSF and other body fluids (pleural, ascitic, peritoneal fluid).The study period was 6 months starting from July 2016 to December 2016 conducted in the Department of Microbiology, Sree Balaji Medical College \& Hospital, Chennai. Appropriate ethical clearance was obtained. It is a prospective study and the data was statistically analyzed. Isolation and identification of P.aeruginosa was done using standard biochemical tests and were subjected to antibiotic susceptibility testing by Kirby - 
Bauer disk diffusion method. The results were interpreted according to Clinical Laboratory Standards Institute (CLSI) guidelines. P.aeruginosa ATCC 27853 was used as control strain.
The susceptibility testing was carried out against the following antibiotics.

Table I: List of Antimicrobial agents tested

\begin{tabular}{|l|c|}
\hline \multicolumn{1}{|c|}{ Antimicrobial agent } & Disc concentration( $\boldsymbol{\mu g}$ /disc) \\
\hline Ceftazidime & 30 \\
\hline Gentamicin & 10 \\
\hline Ciprofloxacin & 5 \\
\hline Aztreonam & 30 \\
\hline Imipenem & 10 \\
\hline Amikacin & 30 \\
\hline Piperacilllin-Tazobactam & $100 / 10$ \\
\hline
\end{tabular}

The pure isolates of Pseudomonas aeruginosa were suspended in $0.5 \mathrm{ml}$ of sterile broth and incubated for 2 hours. The turbidity was matched to $0.5 \mathrm{McFarland}$ standard. The inoculum was spread evenly over the entire surface of the Mueller-Hinton agar plates. This was achieved by swabbing back and front across the agar in three directions. This gives a uniform spread to the entire surface. The antibiotic disc of appropriate potency was applied on to the inoculated plates by means of a sterile forceps. Then, the plates were incubated at $37^{\circ} \mathrm{C}$ for 18 hours in an inverted position. After $18 \mathrm{~h}$ of incubation, the plates were examined and the diameters of zone of inhibition were measured in $\mathrm{mm}^{13}$ and were interpreted as Sensitive, Intermediate \& Resistant according to CLSI guidelines

\section{Result}

Among the total gram negative organism isolated from various clinical samples received Pseudomonas aeruginosa comprised of about $29 \%$. Among the total isolates the maximum number of samples were from swab $\&$ pus $(63 \%)$ next comes urine (8\%), fluid (4\%), blood (3\%) and devices (2\%). Out of the 100 Pseudomonas aeruginosa isolates studied, $87 \%$ were sensitive to Imipenem, 69\% to Amikacin,, $82 \%$ to Piperacillin-Tazobactam, $69 \%$ to Aztreonam, $31 \%$ to third generation cephalosporins, 34\% to Gentamicin \& $47 \%$ to Ciprofloxacin respectively(Fig I).

\section{Fig I: Antibiotic Sensitivity pattern of isolates}

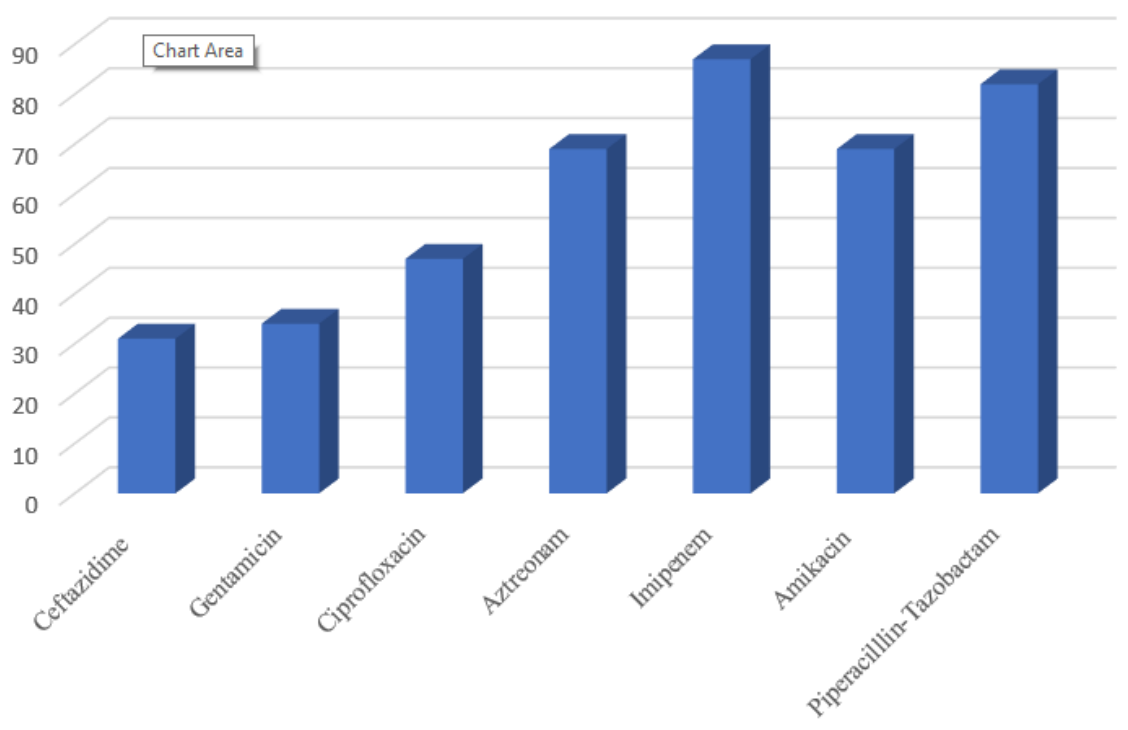

\section{Discussion}

Though antibiotics are used over decades, the resistance genes have equally emerged in bacteria and is spread worldwide causing disease, and also hinder therapy, that leads to progression of infection and death despite therapy. ${ }^{14}$ There is a potential threat because of emergence of multi-drug resistant P. aeruginosa that is reported globally, because of indiscriminate usage of antibiotics. ${ }^{3,15}$

A total of $100 \mathrm{P}$. aeruginosa isolates were taken for sudy. They were isolated, identified and their antimicrobial susceptibility patterns were determined. 
P. aeruginosa isolates were found most sensitive to Imipenem $(87 \%)$ and Piperacillin + Tazobactam $(82 \%)$. About $69 \%$ of isolates were resistant to Ceftazidime. Our results correlated with a study by Vikas Chandra Yadav et al in 2016 where 93\%, 91\%\& 52\% of the P. aeruginosa isolates were sensitive to Imipenem, Piperacillin-Tazobactam \& Ceftazidime respectively. ${ }^{6}$ A similar finding was observed by Patel $\mathrm{H}$ et al which showed a sensitivity of $90 \%$ to Imipenem, $79 \%$ to Piperacillin Tazobactum and $48 \%$ to Ceftazidime. ${ }^{16}$

Another study by Nishi Tiwari et al in 2017, showed resistance pattern of $\mathrm{P}$. aeruginosa strains as follows. Ceftazidime (60\%), Cefepime (52\%), Levofloxacin (49\%), Ticarcillin/clavulanic acid (49\%), Meropenem \& Gentamycin (44\%), Ciprofloxacin (43\%), Amikacin (41\%), Tobramycin (39\%), Netlimycin (36\%), Piperacillin (32\%), Aztreonam (31\%), Piperacillin/tazobactam (26\%), Imipenem (23\%), Doripenem (12\%) \& Gatifloxacin (10\%). ${ }^{5}$ This showed almost similar resistance pattern to Carbapenems \& Piperacillin-tazobactum like our study. Worldwide, about $30 \%$ of strains presented high-level ciprofloxacin resistance. ${ }^{17}$ Our study shows 53\% resistance to ciprofloxacin. A slightly lower resistance rates was seen in another study with $24.1 \%$ to ciprofloxacin. ${ }^{8}$

A recent article published in Saudi Arabia by Ahmed OB in the year 2016 shows a resistance of $28.7 \%$ to Ceftazidime $38.9 \%$ to Piperacillin Tazobactum and $25 \%$ to Imipenem which was comparable to our study. ${ }^{8}$ A study done by Fatima et al 2012 in Pakistan among lower respiratory infection shows a resistance pattern of $24 \%$ to Imipenem, $42 \%$ Piperacillin Tazobactum. This shows the increasing trend of resistance patterns which may be attributed to the increasing usage of drugs for empirical management. ${ }^{18}$ Piperacillin tazobactam resistance among P. aeruginosa has become a threat, this may be attributed to the chromosomal $\beta$-lactamases of P.aeruginosa that show low effectiveness against tazobactam. The rate of resistance to both piperacillin and tazobactum is similar to that for piperacillin alone. ${ }^{16}$

Increase in use of carbapenems further increases resistance rates to carbapenems. A study by C. PlüssSuard in 2013 confirmed a correlation between carbapenem use and carbapenem resistance rates at the hospital and regional levels. ${ }^{19}$ Colistin is one of the lastresort antibiotics for multidrug-resistant organisms.In this study colistin was not included. A study ${ }^{20}$ showed low resistance rate to colistin $(5.5 \%)$ against $\mathrm{P}$. aeruginosa. Resistance to colistin is generally rare, it is seen more in the Mediterranean and South-East Asia (Korea and Singapore).$^{20}$ Colistin is nephrotoxic hence not commonly used.

\section{Conclusion}

Antimicrobial resistance among $\mathrm{P}$. aeruginosa is increasing at an alarming rate. There is deficiency of newer antimicrobial agents against $P$. aeruginosa, this makes studies on the antimicrobial resistance patterns an important task in knowing our status in resistance.Injudicious use of drugs is one of the main reasons for emerging drug resistance among $P$. aeruginosa strains which is responsible for prolonged hospital stay, increased expenditure and adverse clinical consequences. In order to control antimicrobial resistance and to keep it in check it is necessary to practice effective antibiotic policies, frequent surveillance programs and adequate infection control measures.

\section{References}

1. Korgaonkar, Aishwarya et al. "Community Surveillance Enhances Pseudomonas Aeruginosa Virulence during Polymicrobial Infection.'Proceedings of the National Academy of Sciences of the United States of America 110.3 (2013):1059-64. PMC. Web. 5 Dec. 2017.

2. Winn W, Allen S, Janda W, Koneman F, Procop G et al. Color Atlas and textbook of diagnostic microbiology. 6thed. Lippincott Williams and Wilkins (2006),318-20.

3. Ibukun A, Tochukwu N, Tolu O. "Occurrence of ESBL and MBL in clinical isolates of Pseudomonas aeruginosa from Lagos, Nigeria". Journal of American Science (2007),3(4):81-5.

4. Lambert, P A. "Mechanisms of Antibiotic Resistance in Pseudomonas Aeruginosa." Journal of the Royal Society of Medicine 95.Suppl 41(2002):22-6. Print.

5. Nishi Tiwari, Sangita Rajdev, Summaiya Mullan. "Resistance Trends among Pseudomonas aeruginosa Isolates in a Tertiary Care Centre in South Gujarat'. Advances in Microbiology (2017), 7,188-94

6. Vikas Chandra Yadav, Vepada Ravi Kiran, Mahendra Kumar Jaiswal, Khileshwar Singh. "A study of antibiotic sensitivity pattern of Pseudomonas aeruginosa isolated from a tertiary care hospital in South Chhattisgarh ijmsph"( 2017),6(3).

7. Collee J.G., Fraser A.G., Marmion B.P., Simmons A. "Mackie and McCartney Practical Medical Microbiology", 14th edition. Churchill Livingstone, New York, 1996.

8. Ahmed OB.'Incidence and Antibiotic Susceptibility Pattern of Pseudomonas aeruginosa Isolated from Inpatients in Two Tertiary Hospitals". Clin Microbiol (2016)5:248.

9. Gamal F. Gad, Ramadan A. El-Domany, Sahar Zaki, Hossam M. Ashour; "Characterization of Pseudomonas aeruginosa isolated from clinical and environmental samples in Minia, Egypt: prevalence, antibiogram and resistance mechanisms ".Journal of Antimicrobial Chemotherapy, (2007)Volume 60, Issue 5, Pages 101017.

10. Ahmed Bakr Mahmoud, Wafaa Ahmed Zahran, Ghada Rashad Hindawi, Aza Zaghlol Labib and Rasha Galal, "Prevalence of Multidrug-Resistant Pseudomonas aeruginosa in Patients with Nosocomial Infections at a University Hospital in Egypt, with Special Reference to Typing Methods," Journal of Virology \& Microbiology, (2013), Article ID 290047, 13 pages.

11. Biswal, Indu et al. "Incidence of Multidrug Resistant Pseudomonas Aeruginosa Isolated from Burn 
Patients and Environment of Teaching Institution." Journal of Clinical and Diagnostic Research:(2014):DC26-DC29.

12. C. Ding et al," Prevalence of Pseudomonas aeruginosa and antimicrobial-resistant Pseudomonas aeruginosa in patients with pneumonia in mainland China: a systematic review and meta-analysis." International Journal of Infectious Diseases (2016)49:119-28.

13. Benson HJ. "Antimicrobial sensitivity testing: The KirbyBauer method. In: Benson HJ, editor. Microbiological Application: Laboratory Manual in General Microbiology". 7th ed. Boston: McGraw Hill;(1998). p.139-41

14. Istúriz RE, Carbon C.” Antibiotic use in developing countries. Infection Control and Hospital Epidemiology",(2000);21:394-7.

15. Noyal Mariya Joseph, Sheela Devi, P. Shashikala et al. "Changing Trend in the Antibiotic Resistance Pattern of Pseudomonas Aeruginosa Isolated from Wound Swabs of Out-Patients and in-Patients of a Tertiary Care Hospital". Journal of Clinical and Diagnostic Research. (2013) Oct, Vol-7(10):2170-2

16. Patel H, Garala RN. Antibiotic susceptibility pattern of pseudomonas aeruginosa isolated at SSG hospital Baroda. J Res Med Den Sci 2014;2(1):84-7.

17. . Manno G, Cruciani M, Romano L, Scapolan S, Mentasti $\mathrm{M}$, et al. "Antimicrobial use and Pseudomonas aeruginosa susceptibility profile in a cystic fibrosis centre". Int J Antimicrob Agents (2005),25,193-7.

18. Fatima et al. "Antimicrobial susceptibility pattern of clinical isolates of Pseudomonas aeruginosa isolated from patients of lower respiratory tract infections" SpringerPlus (2012), 1:70.

19. Plüss-Suard, C. et al. "Impact of Antibiotic Use on Carbapenem Resistance in Pseudomonas Aeruginosa: Is There a Role for Antibiotic Diversity?"Antimicrobial Agents and Chemotherapy 57.4 (2013): 1709-1713.

20. Bialvaei AZ."Samadi Kafil H Colistin, mechanisms and prevalence of resistance". Curr Med Res Opin (2015) 31:707-721.

How to cite this article: Sindhu Cugati S, Sharanya K, Chitralekha Saikumar C. Antibiotic profile of Pseudomonas aeruginosa strains isolated in a teritiary care centre. Indian J Microbiol Res 2018;5(1):115-118. 